\title{
Concomitant Venous Thromboembolism [VTE] with Cancer and its Effect on Survival in Indian Patients
}

\author{
G Lohith*, Radheshyam Naik, Shekar Patil, Niti Narang Raizada, Amrit Ram, Sudhakar Sampangi, Shivakumar \\ Swamy and Basavalinga Ajai Kumar \\ Healthcare Global Enterprises Limited, Bengaluru, India
}

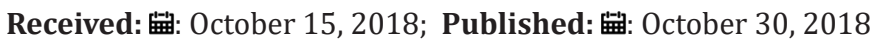

*Corresponding author: G Lohith, Consultant Radiation Oncologist, Healthcare Global enterprises limited, Bengaluru, India

\begin{abstract}
Cancer patients concurrently diagnosed with deep venous thrombosis have a poorer survival. In summary, Concurrent deep venous thrombosis confers a worse prognosis upon cancer patients. Stage at diagnosis, treatment effects, Medical comorbidities could explain differences by cancer site and time frame between deep venous thrombosis diagnosis and cancer outcome and overall survival.
\end{abstract}

Abbreviations: VTE: Venous Thromboembolism; LMWH: Low-Molecular-Weight Heparin; UFH: Unfractionated Heparin; VKA: Vitamin K Antagonists

\section{Introduction}

There has been proven and established relationship between thrombosis and cancer, though the pathophysiological mechanisms remains poorly understood. Numerous studies have shown that cancer multiplies the risk of venous thromboembolism [VTE] by manifold [1]. Cancer increases the risk of thromboembolic syndromes by causing alterations in blood flow; compression of blood flow or invasion of blood vessels by the tumor; activation of coagulation through interactions with platelets, clotting factors and the fibrinolytic system; immobility due to cancer related debility; or endothelial damage due to chemotherapy [2]. Approximately $15 \%$ of all cancer patients will develop thromboembolic syndrome, which is the second leading cause of death in cancer patients [3] and thromboembolic episodes have shown an increase in presence of higher age, medical co-morbidities, immobilization, surgery etc [4]. Patients with thromboembolism, at or just preceding cancer diagnosis, had more advanced disease and lower survival when compared to cancer patients without prior history of thromboembolism [5].

There is substantial biological heterogeneity among various types of cancers, VTE may not be associated with increased rates of death for all cancers. Compression stockings, electrical calf stimulation, intermittent pneumatic compression devices, and venous foot pump devices are used for mechanical thromboprophylaxis. Low molecular weight heparin most frequently used pharmacologic agents as a treatment modality for VTE [6]. Effective prophylaxis and good treatment of VTE has reduced mortality and morbidity, with improved quality of life. Low-molecular-weight heparin [LMWH] is the preferred means for prophylaxis and treatment of VTE. It has largely replaced unfractionated heparin [UFH] and vitamin $\mathrm{K}$ antagonists [VKAs] [7]. We conducted a retrospective study to evaluate the incidence of venous thromboembolism in Indian cancer patients and its effect on the overall survival.

\section{Materials and Methods}

Study of retrospective nature including all adult cancer patients presenting to the oncology ward or day care center at HCG cancer Hospital at Bangalore for diagnosis or treatment of cancer. Adults [>18 years] participants were considered eligible if they received treatment between June, 2010 and August, 2018. They should have initiated and completed all treatment at HCG Hospitals while maintaining a follow-up of atleast 3months post completion of treatment or until death, whichever came first. Excluded were those on anti-platelets agents or anticoagulants, with a previous history of DVT or PE [self-reported], known pro-thrombotic disorders or hypercoagulable states.

Cancer patients treated in inpatient or daycare wards were identified through the discharge summaries and ultrasound reports. 96 eligible patients were included. Medical records were reviewed for demographic and clinical data. Radiological investigations for the study population were also reviewed to make sure that no cases of VTE are missed. Other parameters such as upper limb 
thrombosis, lower limb thrombosis and if prior prophylaxis were received was also noted. The date of diagnosis of VTE and last date of follow-up or up to death were considered for evaluation of overall survival. An exemption from institutional review board [Ethics and scientific committee] was obtained because these data were analyzed without the use of personal identifiers. Since no patients were contacted in this study, informed patient consent was not required.

Statistical Methods: In the current study, proportions of venous thromboembolic episodes were evaluated and the mean overall survival was assessed using Kaplan Meier survival graphs.

\section{Results}

We identified 96 patients with diagnosis of 14 common cancers ranging from head and neck cancers to melanomas of the lower limb with concomitant diagnosis of DVT. Upper limb and lower limb DVTs were seen in 17 patients [17.71\%] and 76 patients [79.71\%] of patients respectively. No significant co-relation was noted to Venous thromboembolic episodes to Age, Sex, Medical co-morbidities, habits [Smoking, tobacco chewing and alcohol intake] and the diagnosis but there was a significant trend showing that DVT was prominent in advanced stages and in metastatic disease $[\mathrm{P}<0.05]$ and patients who have undergone chemotherapy, surgery and radiation had an higher risk of both upper and lower limb venous thromboembolic episodes, though not significant statistically $[\mathrm{P}<0.03]$.Early and prompt primary prophylaxis post diagnosis of DVTs proved to improve survival in the above study. Out of the total 96 patients enrolled into the study, 24 events [Deaths] were observed with a mean survival of $4.5 \pm 0.29$ years (Tables $1 \&$ 2) and (Figure 1).

Table 1.

\begin{tabular}{|c|c|c|c|}
\hline \multirow{2}{*}{ Total } & \multirow{2}{*}{ No of Events } & N & \multicolumn{2}{|c|}{ Censored } \\
\cline { 3 - 4 } & 24 & 72 & $75.0 \%$ \\
\hline 96 & 24 & Percent \\
\hline
\end{tabular}

Table 2.

\begin{tabular}{|c|c|c|c|c|c|c|c|}
\hline \multicolumn{4}{|c|}{ Mean } & \multicolumn{3}{|c|}{ Median } \\
\hline \multirow{2}{*}{ Estimate } & \multirow{2}{*}{ Std. Error } & \multicolumn{2}{|c|}{$\mathbf{9 6 \%}$ Confidence Interval } & \multirow{2}{*}{ Estimate } & \multirow{2}{*}{ Std. Error } & \multicolumn{2}{|c|}{$\mathbf{9 6 \%}$ Confidence Interval } \\
\cline { 3 - 4 } \cline { 6 - 8 } & & Lower Bound & Upper Bound & & Lower Bound & Upper Bound \\
\hline 4.469 & 0.289 & 3.902 & 5.035 & 6.144 & 0.000 & & \\
\hline
\end{tabular}

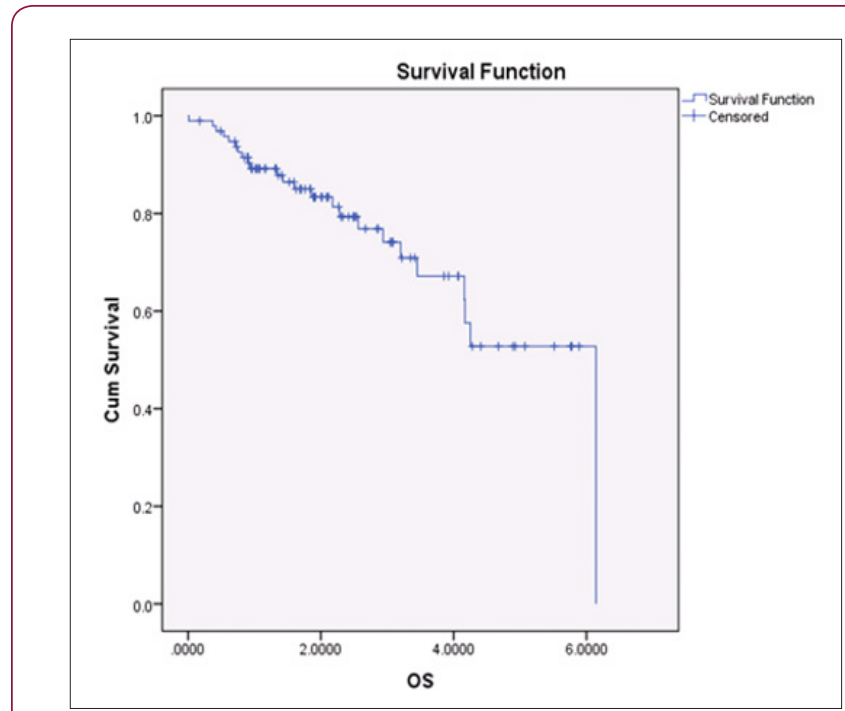

Figure 1.

\section{Comments}

This study enumerates the magnitude of venous thromboembolism after the diagnosis of common cancers on survival outcomes. An important finding of the present study was the strong association between locally advanced and metastatic-stage to venous thromboembolic episodes. The incidence of thromboembolic episodes were highest among patients with either metastatic or lo- co-regionally advanced in the first few months after diagnosis, with the incidence decreasing over time for most cancers. This finding suggests that the biological aggressiveness of the cancer may be the principal risk factor associated with development of thromboembolism and other factors, such as major surgery, chemotherapy, or radiation treatment, contribute to the high incidence of thromboembolism in the advanced stage of disease process as proved in our study. Study findings demonstrate the effect of venous thromboembolism on survival was similar for all the evaluated cancers among patients with localized, regional, or metastatic-stage disease. Further studies are required to determine the reason that venous thromboembolism is so strongly associated with decreased survival.

Sex, age and comorbidities was not a significant predictor of thromboembolism in any of the cancers analyzed in this database. Although a recent study reported that men had a higher risk of recurrent idiopathic venous thromboembolism than women that study specifically excluded cancer patients [8]. VTE's are common in cancer patients undergoing surgery, chemotherapy and radiotherapy and the above study proves that the risk of VTE's are heightened with aggressive treatment strategies availed by the cancer patients and this has been noted in Khorana et.al. [9]. Early prophylaxis in cancer patients improved survival and this co-relates with the standard norm of early prophylaxis with low molecular weight heparins leading to better control and overall survival [10]. There are several limitations to this study; Inclusion 
of the 96 patients who presented with venous thromboembolism and were concurrently diagnosed with cancer could be viewed as inflating the incidence of thromboembolism among cancer cases. A comparative arm would be appropriate to evaluate the burden of venous thromboembolism in the Indian cancer population.

\section{Summary}

This study used Indian cancer based data to estimate the incidence, time course of venous thromboembolism among patients with cancer. The presence of venous thromboembolism was seen as a significant predictor of death for all cancer types analyzed, and this was true for patients with localized, regional, or metastaticstage disease. These findings identify potential populations that might be considered for thromboprophylaxis and in-turn improve the outcomes of cancer patients diagnosed with VTE's.

\section{References}

1. Stein PD, Beemath A, Meyers FA, Skaf E, Sanchez J, et al. (2006) Incidence of venous thromboembolism in patients hospitalized with cancer. Am J Med 119(1): 60-68.

2. Green KB, Silverstein RL (1996) Hypercoagulability in cancer. Hematology/Oncology Clinics 10(2): 499-530.

3. Donati MB (1994) Cancer and thrombosis. Haemostasis 24(2): 128-131.

ISSN: 2574-1241

DOI: $10.26717 / B J S T R .2018 .10 .001980$

G Lohith. Biomed J Sci \& Tech Res

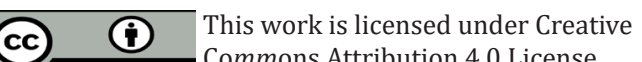

Submission Link: https://biomedres.us/submit-manuscript.php
4. Heit JA, Silverstein MD, Mohr DN, Petterson TM, O'Fallon WM, et al. (2000) Risk factors for deep vein thrombosis and pulmonary embolism: A population-based case-control study. Arch Intern Med 160(6): 809815.

5. Sorensen HT, Mellemkjaer L, Olsen JH, Baron J (2000) Prognosis of cancers associated with venous thromboembolism. N Engl J Med 343(25): 1846-1850.

6. Wells PS, Lensing AW, Hirsh J (1994) Graduated compression stockings in the prevention of postoperative venous thromboembolism. A metaanalysis. Arch Intern Med 154(1): 67-72.

7. Lee AY, Levine MN, Baker RI, Bowden C, Kakkar AK, et al. (2003) Randomized Comparison of Low-Molecular-Weight Heparin versus Oral Anticoagulant Therapy for the Prevention of Recurrent Venous Thromboembolism in Patients with Cancer (CLOT) Investigators. Lowmolecular-weight heparin versus a coumarin for the prevention of recurrent venous thromboembolism in patients with cancer. $\mathrm{N}$ Engl J Med 349(2):146-153.

8. Moores L, Bilello KL, Murin S (2004) Sex and gender issues and venous thromboembolism, Clin Chest Med 25(2): 281-297.

9. Alok A. Khorana, Gregory C. Connolly (2009) Assessing Risk of Venous Thromboembolism in the Patient with Cancer, J Clin Oncol 27(29): 48394847.

10. Dolovich LR, Ginsberg JS, Douketis JD, Holbrook AM, Cheah G, et al. (2000) A meta-analysis comparing low-molecular-weight heparins with unfractionated heparin in the treatment of venous thromboembolism. Arch Intern Med 160(2): 181-188.

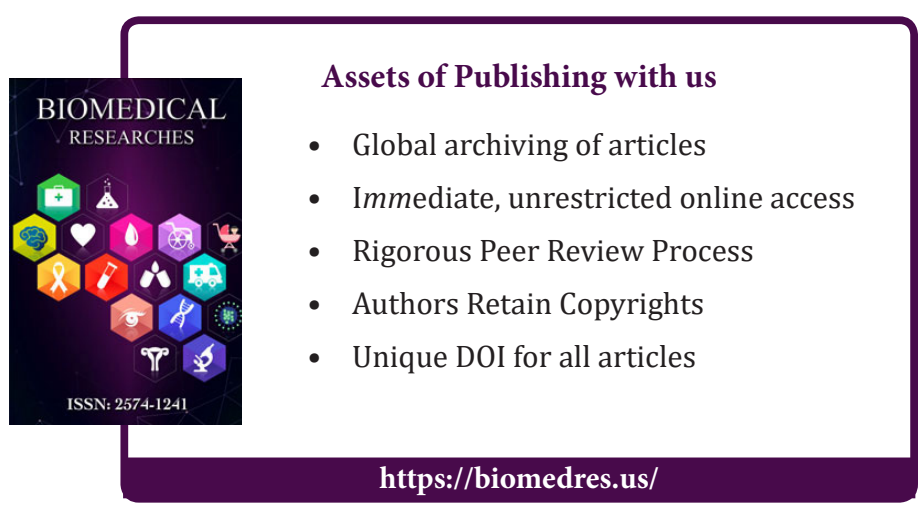

